\title{
HSA-Based Optimal Allocation and Sizing of Shunt Compensators Considering Cable Aging Constraint and Load Variations
}

\author{
H. Karami ${ }^{1}$, M.J. Sanjari ${ }^{1}$, A. Tavakoli ${ }^{1}$, G.B. Gharehpetian ${ }^{1}$, M.S.A. Hejazi ${ }^{2}$ \\ ${ }^{1}$ Electrical Engineering Department \\ Amirkabir University of Technology \\ Tehran, Iran \\ Phone/Fax number: +982166406469, e-mails: hkp6785@gmail.com,m_j_sanjari@aut.ac.ir, atrin.tavakoli@aut.ac.ir, \\ grptian@aut.ac.ir \\ ${ }^{2}$ Electrical Engineering Department \\ University of Kashan \\ Kashan, Iran \\ Phone/Fax number: +983614444772, e-mails: mhejazi@kashanu.ac.ir
}

\begin{abstract}
Aging of power system equipments can be caused by erosion of metallic structures such as cable sheathing. Higher system risk due to higher failure probability and possible system damage following the endof-life failure, are the direct consequence of cable aging. One of the solutions to solve this problem is the reduction of the cables current flow using different solutions in the power system network. In this paper, an HSA-Based optimal placement and sizing of shunt compensators is proposed to reduce the current flow. The IEEE RTS 24-Bus network is selected to verify the proposed algorithm with and without considering load variations during a year. The obtained results show the possibility of slowing of the cable aging in the studied network using shunt compensators.
\end{abstract}

\section{Keywords}

Cable aging, Shunt compensator, HSA, Load variations, IEEE RTS 24-Bus.

\section{Introduction}

There may be different causes of aging for different kinds of equipment. Aging can be caused by erosion of metal structures (such as underground cable sheathing), fatigue damage of mechanical parts (such as generators and motors), or insulation deterioration of electrical components (such as transformers and reactors). Like human beings, any equipment will experience its wear-out stages, normal operation, and infancy. As a piece of equipment ages, it fails more frequently, eventually reaches its end of life, and needs longer time to repair. Higher system risk due to higher failure probability and possible damage of system following the end-of-life failure is the consequence of cable aging. Utilities normally carry out regular inspections and preventative overhauls. The maintenance activities can extend the life of equipments but could be costly for equipments at their end-of-life stage. Therefore, a compromise between replacement and maintenance must be carefully considered.

Recently many studies have been conducted on cable aging in networks [1-6]. In [5], the effect of the cable aging has been considered in optimal allocation and sizing of DGs in power system. In this paper, Harmony Search Algorithm (HSA) is used to find the optimal allocation and sizing of shunt compensators in order to solve the cable aging in the power system network. The effectiveness of the proposed algorithm is demonstrated through IEEE RTS 24-Bus standard test network simulations with and without considering the load variations during a year.

\section{Cable Aging Process and Maintenance}

The probability of end-of-life failures increases with cable aging and aging process can be slowed through the maintenance activities. There are two kinds of maintenance: corrective and preventative. The corrective maintenance is a repairing activity after a cable failure and deals with a repairable failure. The preventative maintenance is an inspection or overhaul activity before a failure. The major goal of the preventative maintenance is to reduce deterioration and prolong the lifetime of the 
cable, and it addresses both end of life and repairable failures. The economic or usable value of the cable is reduced as it ages, and preventative maintenance activities can delay the aging process. The relationship between the preventative maintenance, time, and value is shown in Figure 1. It can be seen that maintenance can recover a part of the lost value caused by the deterioration in the aging process. However, although maintenance can slow the aging, it cannot fully stop it.

There are two methods for implementing preventative maintenance: predictive and regular. The predictive maintenance has been implemented in some utilities in the past 10-15 years and addressed in the power industry. This method needs an assessment process including calculations and mathematical modeling, condition monitoring, and appropriate criteria. The regular maintenance is planned for fixed intervals whiles the predictive maintenance is undertaken as needed. The regular maintenance is based on the experience of the maintenance personnel or manufacturer's specification and is the course most widely used by utility. This method is easy and simple to perform but may result in either higher costs (doing unnecessary maintenance activities) or high system risk (not doing in time necessary maintenance activities).

As shown in Fig. 1, if the ratio of the current flow to the nominal current in each branch reduces, the cable life will be prolonged. In this paper, the cable aging is modeled in the objective function. In other words, the following equation should be minimized:

$C_{1} \sum_{i=1}^{N_{\text {branch }}} \frac{\text { No minal life of cable } i}{\text { Prolonged life of cable } i \text { after compensation }}$

In this paper, it is assumed that the compensator is a shunt device. The costs of the reactive power injection should be considered in the objective function. Therefore, the objective function should be defined as follows:

$$
\begin{aligned}
& C_{1} \sum_{i=1}^{N_{\text {bman }}} \frac{\text { Nominal life of cable } i}{\text { Prolonged life of cable } i \text { after compensation }} \\
& +\mathrm{C}_{2} \sum_{i=1}^{N_{\text {bus }}} Q_{\text {compensator }^{p u}}
\end{aligned}
$$

where, $C_{1}$ and $C_{2}$ are weighting factors and $Q_{\text {compensator }}^{p u}$ is the cost of the reactive power injection. In this paper, we have $C_{1} / C_{2}=0.5$, because the minimization of the injected reactive power, i.e. $Q_{\text {compensator }}^{p u}$, is more important. The relationships between voltage profile and network loss have been studied in [7] considering compensators sizing and placement. The goal of this paper is considering the cable aging in this issue. Therefore, in order to focus on cable aging, it is assumed that the effects of network loss and voltage profile are neglected through the cost function calculation. In the next section, an optimization algorithm will be described and used for finding the optimal number, injected reactive power values and location of shunt devices.

\section{Harmony Search Algorithm}

HSA has been extracted and developed from the music improvisation process, in which music players improvise their instrument pitches to get better harmony [8-14]. The procedure of HSA is described in the next five steps:

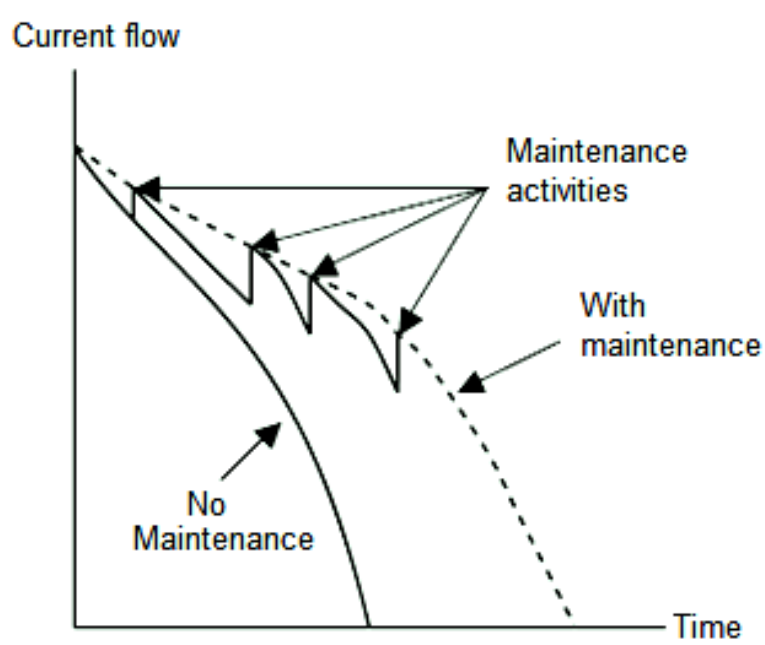

Fig. 1. Relationship among time, preventative maintenance, and current value

Step 1: Initialize the algorithm parameters and problem The optimization of the problem is specified as follows: $\min \{f(x) \mid x \in X\}$

subject to: $\quad g(x) \geq 0$ and $h(x)=0$

where the inequality constraint function is $\mathrm{g}(\mathrm{x})$, the objective function is $\mathrm{f}(\mathrm{x})$, and the equality constraint function is $\mathrm{h}(\mathrm{x})$. The set of each decision variable is $\mathrm{x}$, and the set of the possible range of values for each decision variable is $\mathrm{X}$, that is $X_{i, \min } \leq x_{i} \leq X_{i, \max }$, where, $X_{i, \max }$ and $X_{i, \min }$ are the upper and lower bounds for each decision variable, respectively. In the first step, the HSA parameters are also specified. These are the number of solution vectors in the harmony memory $(H M)$, or the harmony memory size $(H M S)$, number of decision variables $(N)$, harmony memory considering rate $(H M C R)$; pitch adjusting rate $(P A R)$ and the number of improvisations $(N I)$, or stopping criterion. Similar to the genetic pool in GA the $H M$ is a matrix, where all of the solution vectors are stored in it [11]. Here, $P A R$ and $H M C R$ are parameters that both of them are defined in Step3 and used to improve the solution vector.

Step 2: Initialize the harmony memory

The $H M$ matrix will be filled with as many randomly generated vectors as the $H M S$, in Step 2, as follows:

$$
H M=\left[\begin{array}{ccccc}
x_{1}^{1} & x_{2}^{1} & \ldots & x_{N-1}^{1} & x_{N}^{1} \\
x_{1}^{2} & x_{2}^{2} & \ldots & x_{N-1}^{2} & x_{N}^{2} \\
\vdots & \vdots & \vdots & \vdots & \vdots \\
x_{1}^{H M S-1} & x_{2}^{H M S-1} & \ldots & x_{N-1}^{H M S-1} & x_{N}^{H M S-1} \\
x_{1}^{H M S} & x_{2}^{H M S} & \ldots & x_{N-1}^{H M S} & x_{N}^{H M S}
\end{array}\right]
$$

\section{Step 3: Improvise a new harmony}

The new harmony vector is generated based on three rules: random solution generation, pitch adjustment and memory consideration. Creating a new harmony is called 'improvisation' [10]. The value of the first decision variable $x_{1}$ ' for the new vector in the harmony memory consideration is chosen from any value in the specified rang of $H M\left(\mathrm{x}_{1}{ }^{1}\right.$ to $\left.x_{l}{ }^{H M S}\right)$. In the same approach, values of other decision variables $\left(x_{2}, \ldots, x_{N}{ }^{\prime}\right)$ are chosen. (1-HMCR), which varies between 0 and 1 , is the selection rate of one 
value from the possible range of values randomly, while $H M C R$ is the rate of choosing one value from the historical values stored in the $H M$ as follows:

$$
\left.\begin{array}{ll}
x_{i}^{\prime} \in\left\{x_{i}^{1}, x_{i}^{2}, \ldots, x_{i}^{\text {HMS }}\right\} \text { with probability HMCR } \\
x_{i}^{\prime} \in X_{i} & \text { with probability }(1-H M C R)
\end{array}\right\} \rightarrow x_{i}^{\prime}
$$

For example, an HMCR of 0.60 indicates that the decision variable will be chosen from historically stored variables in the $H M$ with the $60 \%$ probability or from the entire possible range with the $40 \%$ probability. The elements that get by the memory consideration is examined to determine whether or not it should be pitch-adjusted. This operation uses the PAR parameter, which has the rate of pitch adjustment, as follows:

$$
\left.\begin{array}{l}
Y e s \text { with probability } P A R \\
\text { No with probability }(1-P A R)
\end{array}\right\}
$$

The rate of doing nothing with the decision variable value is set by the value of $(1-P A R)$. If the pitch adjustment decision for $x_{i}$ ' is Yes, $x_{i}$ ' is replaced as follows:

$$
x_{i}^{\prime} \leftarrow x_{i}^{\prime} \pm \operatorname{rand}() * b_{w}
$$

where, $b_{w}$ is the distance bandwidth that in step 1 is set and rand() is a number that randomly varies between 0 and 1 .

In Step 3, the random selection or pitch adjustment and $H M$ consideration is applied to each variable of the New Harmony Vector $(N H V)$ in turn.

\section{Step 4: Update harmony memory}

If the $N H V$ is better than the worst harmony in the $H M$, the new harmony vector is included in the $H M$ and the existing worst harmony is excluded.

Step 5: Check stopping criterion

If the maximum number of improvisations is reached, the computation is terminated. Otherwise, Steps 3 and 4 are repeated.

\section{Simulation Results}

The IEEE RTS 24-bus test system, used in this paper, is shown in Fig. 2. The objective function is defined in equation 2 such that 0.95 p.u. $\leq V_{i} \leq 1.05$ p.u. $\quad(1 \leq \mathrm{i} \leq 24)$, $N_{\text {branch }}=38$ and $N_{\text {bus }}=24$. Every row of the $H M$ matrix has 48 elements, the first 24 elements of rows indicate the busses that the shunt compensator should be installed there, and the second 24 elements show the reactive power that should be injected by the compensator. Table 1 shows a row of the $H M$ matrix.
It should be noted that the first 24 elements are 0 or 1 and other elements are selected between $0.05 \mathrm{pu}$ and $0.25 \mathrm{pu}$. In each iteration, HSA finds a better solution that leads to a less value of objective function.

These values are saved in the $H M$ matrix. This network is simulated with and without considering variations in the loads during a year. Without considering the load variations, the value of the objective function is shown in Fig. 3 for 100000 iterations. Figs. 3 and 4 show the minimum and mean values of objective function versus the number of iterations, respectively. These two figures show the acceptable convergence and performance of the HSA for this problem.

According to the final results of HSA, the shunt compensators should be placed at buses 8 and 22. The setting of the injected reactive power at buses 8 and 22 are set to 0.2274 pu and $0.2116 \mathrm{pu}$, respectively.

In order to considering the load variations, the reactive and active power of the load at one bus (bus 18 in this simulation) varies with a Gaussian distribution through Monte Carlo simulation [15]. For each vector of $H M$ matrix, the objective function is calculated and the mean of load variations is shown as the results. The output of the objective function considering load variations is shown in Fig. 5 for 10000 iterations. Figs. 5 and 6 show the minimum and mean values of objective function versus the number of iterations, respectively. These two figures show the acceptable convergence and performance of the HSA for this case, as well.

According to the final results of HSA in this case, the compensators should be placed at buses 7,8,11 and 23. The setting of the injected reactive power at buses 7,8,11 and 23 are set to $0.2326 \mathrm{pu}, 0.1845 \mathrm{pu}, 0.0836 \mathrm{pu}$ and $0.2343 \mathrm{pu}$, respectively. It is obvious that the optimization procedure results in better solution (lower value of the objective function), if the load variation does not considered. But considering the load variation has more real results.

\section{Conclusion}

In this paper, the optimal allocation and sizing of shunt compensators has been proposed based on HSA to reduce the cable aging. As shown in the results, the IEEE RTS 24bus system with and without load variations, has been used to show the importance of this study. The values of the injected reactive power and busses for the installation of compensators have been determined in order to slow the cable aging.

Table I. Each row of $H M$ matrix

\begin{tabular}{|c|c|c|c|c|c|c|c|}
\hline $\begin{array}{c}\text { compensator at } \\
\text { bus } 1(0 \text { or } 1)\end{array}$ & $\begin{array}{c}\text { compensator at } \\
\text { bus } 2(0 \text { or } 1)\end{array}$ & $\ldots$ & $\begin{array}{c}\text { compensator at bus } \\
24(0 \text { or } 1)\end{array}$ & $Q_{\text {injecte }}^{1}$ & $Q_{\text {injecte }}^{2}$ & $\ldots$ & $Q_{\text {injecte }}^{24}$ \\
\hline
\end{tabular}




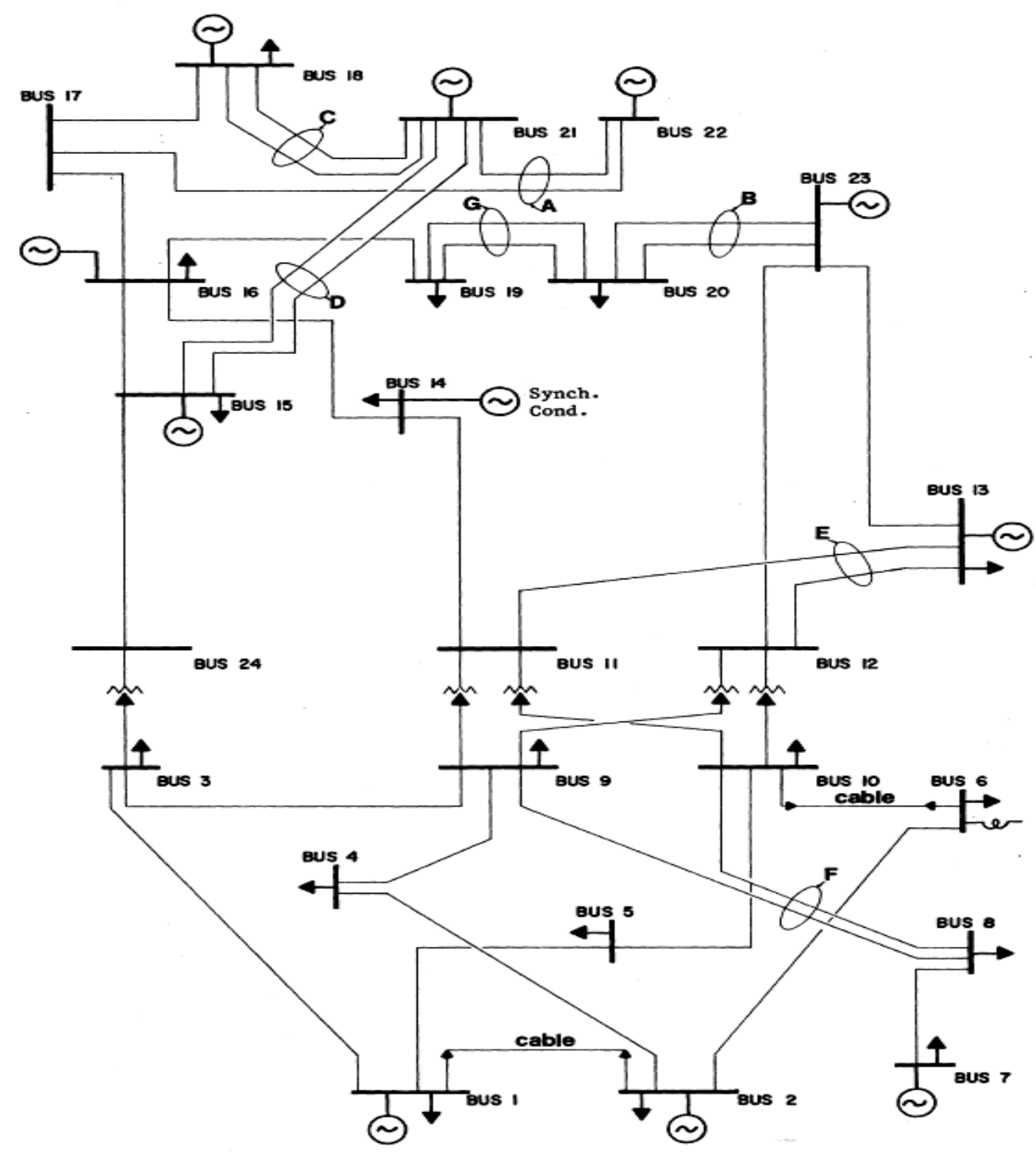

Fig. 2. IEEE RTS 24-bus test system

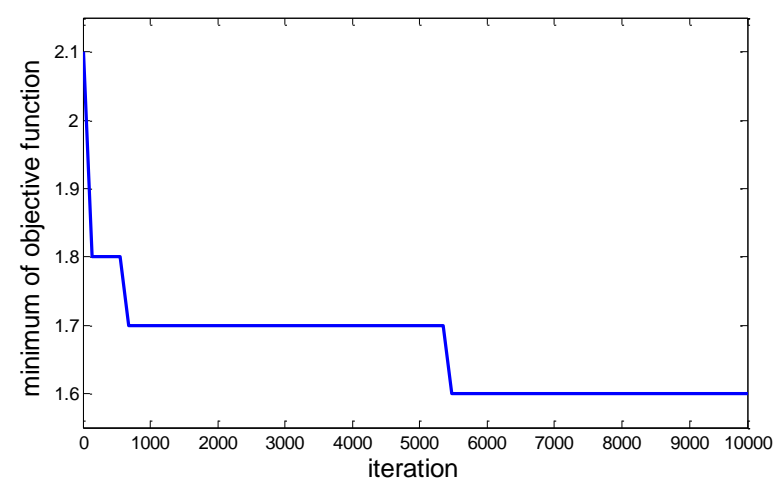

Fig. 3. Minimum value of $H M$ matrix versus number of iterations (without considering the load variation)

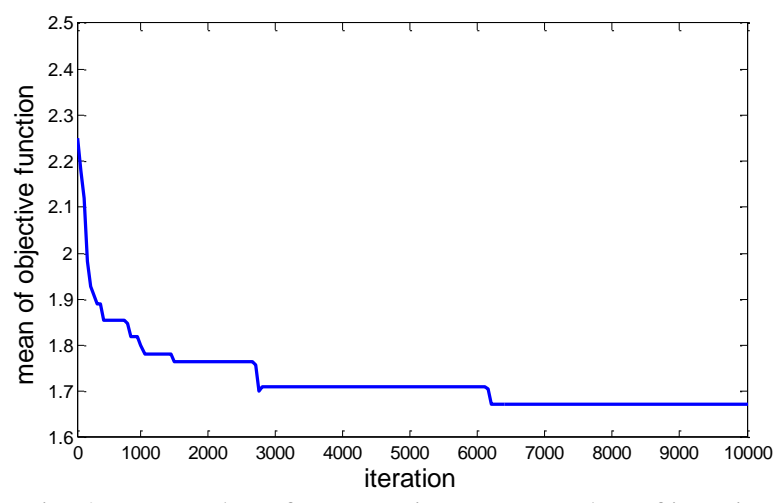

Fig. 4. Mean value of $H M$ matrix versus number of iterations (without considering the load variation) 


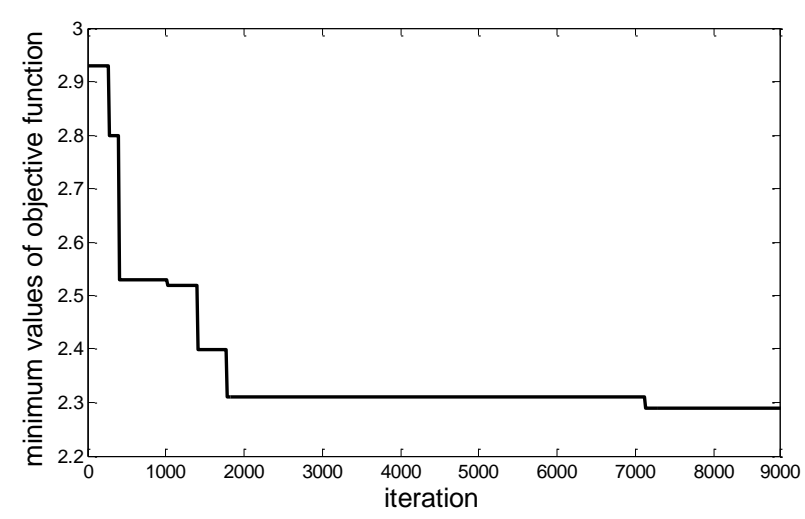

Fig. 5. Minimum value of $H M$ matrix versus number of iterations (with considering the load variation)

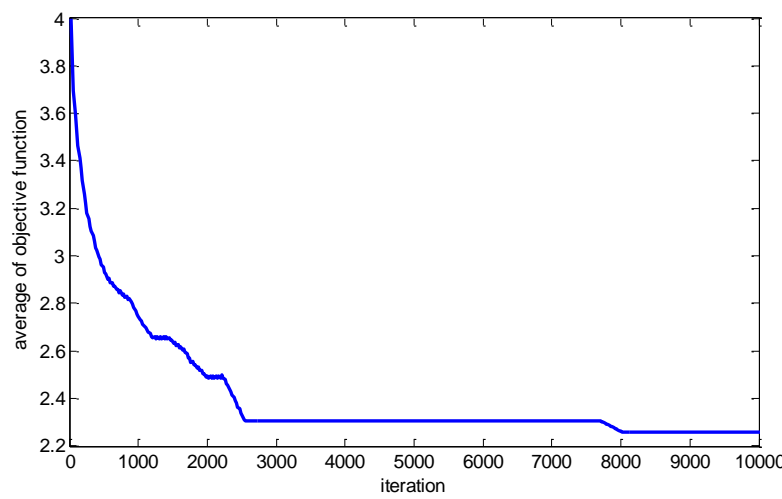

Fig. 6. Mean value of $H M$ matrix versus number of iterations (with considering the load variation)

\section{References}

[1] R.A. Hartlein, V.S. Harper, H.W. Ng, "Effects of Voltage Impulses on Solid Dielectric Cable Life", Power Engineering Review, IEEE, vol. 9, pp. 39-40, 1989.

[2] Lyle, R.; "Effect of testing parameters on the outcome of the accelerated cable life test", Power Delivery, IEEE Transactions on, vol. 3, pp. 434-439, 1988.

[3] Emin, Z.; Basak, P.K.; Ferguson, C.; "Simulation studies to improve design for midlife $275-\mathrm{kV}$ cable refurbishment", Power Delivery, IEEE Transactions on, vol. 18, pp. 679683, 2003.

[4] Robertson, J.; Varlow, B.R.; "The use of non linear permittivity fillers for the purposes of stress grading within cables", Properties and Applications of Dielectric Materials, 2003. Proceedings of the 7th International Conference on, vol.3 pp. 1210-1213, 2003.

[5] H. Karami, M.J. Sanjari, A. Tavakoli, G.B. Gharehpetian, "HSA-Based Optimal Allocation of DGs Considering Cable Aging Constraint", 2nd International Conferences on Renewable Energy and Distributited Generated (ICREDG), Tehran, Iran, 06-08 March, 2012.

[6] Hajagos, J.T.; "Planning response to increase system reliability of the aging T\&D infrastructures", Power Engineering Society Summer Meeting, 2000. IEEE, vol. 3, pp. 1500-1506, 2000.

[7] R. Sirjani, A. Mohamed, H. Shareef, "Optimal allocation of shunt Var compensators in power systems using a novel global harmony search algorithm", International Journal of Electrical Power \& Energy Systems, Vol. 43 (1), December 2012, pp. 562-572.

[8] Zong Woo Geem, "Novel derivative of harmony search algorithm for discrete design variables Environmental Planning and Management Program", Johns Hopkins
University,729 Fallsgrove Dr. \#6133, Rockville, MD 20850, USA.

[9] H. Karami, M. J. Sanjari, A. Tavakoli and G. B. Gharehpetian, "Optimal Scheduling of Residential Energy System Including CHP System and Storage Device", Electric Power Components and Systems, Volume 41, Issue 8, April 2013, pages 765-780.

[10] M. J. Sanjari, S. H. Fathi, G. B. Gharehpetian and A. Tavakoli, "HSA-Based Optimal Placement of Shunt FACTS Devices in the Smart Grid Considering Voltage Stability", 2nd Iranian Conference on Smart Grids, ICSG 2012, Tehran, Iran, May 23-24, 2012.

[11] J. H. Holland, Adaptation in Natural and Artificial Systems. Ann Arbor, MI: University of Michigan Press, 1975.

[12] KS. Lee, ZW. Geem, "a new structural optimization method based on the harmony search algorithm", Computer Structure, Vol. 82, pp. 781-98, 2004.

[13] ZW. Geem, JH. Kim, GV. Loganathan, "harmony search optimization: application to pipe network design", International Journal of Modeling and Simulation, Vol., 22, pp.125-33, 2002.

[14] ZW.Geem, C. Tseng and Y. Park, "harmony search for generalized orienteering problem: best touring in China", Springer Lecture Notes for Computer Science, Vol. 341, pp.741-50, 2005.

[15] M. J. Sanjari, O. Alizadeh Mousavi and G. B. Gharehpetian, "Assessing the Risk of Blackout in the Power System Including HVDC and FACTS Devices", International Transactions on Electrical Energy Systems, Vol. 23, Issue 1, Jan. 2013, pages 109-121. 